\title{
Taylor-SPH vs Taylor-Galerkin for shock waves in viscoplastic continua
}

\author{
Mokhtar Mabssout* — María Isabel Herreros** \\ * Equipe Matériaux et Mécanique des Structures \\ Faculté des Sciences et Techniques de Tanger \\ BP 416, Tanger, Maroc \\ m.mabssout@fstt.ac.ma \\ ** Escuela Técnica Superior de Ingeniería Informática \\ Universidad Rey Juan Carlos (URJC) \\ Madrid, Espagne \\ isabel.herreros@urjc.es
}

\begin{abstract}
A new time discretization scheme with a corrected SPH is presented. The time discretization has been carried out by means of a Taylor series expansion in two steps. In order to avoid numerical instabilities, two different sets of particles have been considered in the time discretization, and a Lagrangian kernel has been used for the spatial approximation. The Lagrangian kernel and its gradient have been corrected to satisfy the consistency conditions. This new method is applied to solve the propagation of shock waves in elastoviscoplastic media and the results are compared with those obtained with a similar time discretization scheme within the frame of FEM. The proposed method is shown to be stable and robust. Numerical dispersion and diffusion are minimized and only a reduced number of particles is required to obtain reasonably accurate results.

RÉSUMÉ. Un nouveau schéma de discrétisation temporelle avec la méthode sans maillage SPH est présenté. La discrétisation dans le temps est effectuée à l'aide d'un développement de Taylor en deux étapes. Pour éviter les instabilités numériques, deux groupes de particules ont été utilisés pour la discrétisation temporelle et une fonction noyau lagrangienne a été utilisée pour l'approximation spatiale. La fonction noyau lagrangienne et son gradient ont été corrigés pour satisfaire les conditions de consistance. Cette nouvelle méthode a été utilisée pour résoudre le problème de propagation d'ondes de choc dans un milieu élastoviscoplastique. Les résultats ont été comparés avec ceux obtenus par MEF utilisant le même schéma de discrétisation dans le temps. La méthode proposée est stable et robuste. Les problèmes de dispersion et de diffusion numériques sont réduits au minimum et seulement un nombre réduit de particules est nécessaire pour obtenir des résultats précis.
\end{abstract}

KEYWORDS: Taylor-SPH, Taylor-Galerkin, shock wave, viscoplastic, FEM.

MOTS-CLÉS : Taylor-SPH, Taylor-Galerkin, onde de choc, viscoplastique, MEF.

DOI:10.3166/EJCM.20.281-308 C 2011 Lavoisier, Paris 


\section{Introduction}

Mesh based numerical methods such as Finite Difference Method (FDM) and Finite Element Method (FEM) have been widely used to solve Solid Mechanics problems.

Despite of their great success, mesh based numerical methods suffer from some difficulties when dealing with problems where large deformations, discontinuities and crack propagation are involved.

To overcome these difficulties, some meshfree methods were developed in the past. They are based on constructing approximating functions at arbitrary discrete points in the domain, and, as a consequence, the need for a mesh is eliminated. One example of meshfree method is the Smoothed Particle Hydrodynamics (SPH). The SPH method was developed in 1977 by Lucy (1977) and Gingold and Monaghan (1977) to be applied in Astrophysics. In 1991, it was extended to solve problems in Solid Mechanics where traditionally FEM fails (Libersky et al., 1991).

It is well known that the original SPH method suffers from tensile instabilities (Swegle et al., 1995) and lack of consistency (Belytschko et al., 1998). Over the past years, different corrections have been introduced to improve the accuracy of the SPH solution. Liu et al., (1995) proposed a correction function that restores the first order completeness of the kernel function. Some years later, Randles and Libersky (1997) developed a transformation of the gradient that provides the correct values of the strains for linear fields; this normalization of the gradient is a generalization of the work proposed before by Johnson and Beissel (1996).

To solve the tensile instability, (Dyka et al., 1995; 1997) introduced the stress point method into SPH. This approach was later extended to higher dimensions by Randles and Libersky (2000). Belytschko et al., (2000) showed that the stress point technique stabilizes SPH by removing the instability that arises due to rank deficiency, i.e. spurious singular modes, while tensile instabilities can be avoided by using a Lagrangian kernel. Other recent studies to improve the convergence of the SPH method can be found in (Belytschko et al., 1998; 1996; Bonet et al., 2000; Krongauz et al., 1997; Monaghan, 1988; Rabczuk et al., 2004; Xiao et al., 2005).

Modelling of shock waves propagation in solids is most demanding area since a wide variety of problems is encountered. Even in the linear range, the short wavelengths are subjected to numerical damping and dispersion. The shocks are smoothed and leading or trailing oscillations appear. The situation is more involved in the non-linear regime, where strain can localize along shear bands which may be affected by the mesh if FEM is used in the analysis.

The key advantage of using SPH is that the absence of a mesh enables it to deal with larger local distortion than Finite Element Methods, and therefore phenomena such as fracture, and other material instabilities are more easily modelled. In addition 
to that, mesh dependence problems such as mesh alignment or mesh size dependence, are solved in a straightforward manner, given the meshfree nature of the method.

Several schemes using SPH for wave and shock propagation in solids can be found in the literature, in particular with applications to impact on plates and interaction fluid-structure (Caleyron et al., 2009; Potapov et al., 2009; ChuzelMarmot et al., 2008; Maurel et al., 2008).

Nevertheless, the SPH method still presents several difficulties when dealing with dynamics and shock wave propagation: numerical damping, depending on relative wavelengths, and numerical dispersion, consisting of short waves propagating faster or slower and causing oscillations close to the front.

The authors of the present paper have published in previous works some different alternatives to solve the propagation of shock waves in solids using FEM (Mabssout $e t$ al., 2003a) (Mabssout et al., 2003b) (Mabssout et al., 2006). In Solid Dynamics, the classical approach of numerical analysis is based on a displacement formulation using the Newmark scheme. It is well established that the Newmark family of methods is not suitable to solve discontinuous phenomena, and many efforts have been devoted in the past to solve this problem (Krenk et al., 2005; Krenk, 2006; Li et al., 2003; Simo et al., 1992; Laursen, 2002; Réthoré et al., 2005). The shortcomings of the classical approach can be classified in: (a) Numerical damping and numerical dispersion; (b) In classical displacement formulations low-order elements cannot be used as they are not accurate and the results depend on mesh alignment and mesh size. To solve the problems mentioned in (b) the authors have proposed in previous works a numerical model formulated in terms of stress and velocity based on a Taylor-Galerkin scheme. This method has been proved to perform well for localized failure of viscoplastic materials of Von Mises type. It was shown that low-order elements, such as linear triangles, exhibited an excellent behaviour and reasonably numerical diffusion and dispersion.

In this paper, a new numerical method for solving the propagation of shock waves in elasto-viscoplastic media is developed. It uses a two-steps time discretization algorithm by means of a Taylor series expansion and a corrected SPH method for the spatial discretization. In order to avoid numerical instabilities, two different sets of particles are considered to perform the time discretization and a Lagrangian kernel is used. Both, Lagrangian kernel and its gradient, are corrected to satisfy the consistency conditions.

The purpose of this paper is to show how the proposed time discretization algorithm combined with a corrected SPH provides solutions of more accuracy than those obtained with the methods proposed in the past within the frame of the Finite Element Method.

The paper is organized as follows. First, the governing equations for dynamic problems in viscoplastic media are given in terms of stress and velocity. In Section 3, equations are discretized using the proposed Taylor-SPH method. To assess the performance of the proposed method, some numerical applications in 1D 
and 2D are described in Section 4. The results are compared to analytical solutions and to numerical results obtained with FEM.

\section{Governing equations}

The governing equations are written in terms of stress and velocity as follows:

- Balance of momentum equation:

$$
\nabla \boldsymbol{\sigma}=\rho \frac{\partial \mathbf{v}}{\partial t}
$$

where $\boldsymbol{\sigma}$ is the stress tensor, $\rho$ is the density and $\mathbf{v}$ is the velocity vector.

- Constitutive equation: It will be assumed that the material behaviour can be described by Perzyna's viscoplastic law (Perzyna , 1966):

$$
\frac{\partial \boldsymbol{\sigma}}{\partial t}=\mathbf{D}^{e}:\left(\frac{\partial \boldsymbol{\varepsilon}}{\partial t}-\frac{\partial \boldsymbol{\varepsilon}^{v p}}{\partial t}\right)
$$

being $\mathbf{D}^{e}$ the elastic constitutive tensor and $\varepsilon$ the strain tensor; the superindex $v p$ refers to the viscoplastic component of the strain tensor given by:

$$
\frac{\partial \boldsymbol{\varepsilon}^{v p}}{\partial t}=\gamma \mathbf{m}\langle\phi(F)\rangle
$$

In above, $<_{.>}$are the Macaulay brackets, the scalar $\gamma$ is the fluidity parameter, and $\mathbf{m}$ characterizes the direction of the plastic flow.

Among several alternatives for the function $\phi(F)$, it will be used here

$$
\phi(F)=\left(\frac{F-F_{0}}{F_{0}}\right)^{N}
$$

being $N$ a model parameter and $F$ a function describing a convex surface in the stress space. The value $F_{0}$ characterizes the stress level below which no flow occurs. In the case of a Von Mises material, $F$ is taken as the effective deviatoric stress $\bar{\sigma}$

$$
F=\bar{\sigma}=\sqrt{\frac{3}{2} s_{i j} s_{i j}}
$$

being $\mathbf{s}$ the deviatoric stress tensor. The size of the yield surface $F_{0}=\overline{\sigma_{0}}$ will vary according to a suitable hardening/softening law. Here it will be assumed a linear dependence on the equivalent deviatoric plastic strain $\bar{\varepsilon}^{v p}$.

$$
\frac{\partial \bar{\sigma}_{0}}{\partial t}=H \frac{\partial \bar{\varepsilon}^{v p}}{\partial t}
$$

where $H$ is the hardening/softening modulus. 
It will be also assumed an associated flow rule, such that: $\mathbf{m}=\frac{\partial F}{\partial \boldsymbol{\sigma}}$

Finally, to express the rate of stress in terms of the gradient of velocity, the following kinematic relation is used:

$$
\frac{\partial \varepsilon_{i j}}{\partial t}=\frac{1}{2}\left(\frac{\partial v_{i}}{\partial x_{j}}+\frac{\partial v_{j}}{\partial x_{i}}\right)
$$

In two dimensional situations, stress and strain are represented as

$$
\begin{aligned}
& \boldsymbol{\sigma}=\left(\sigma_{11}, \sigma_{22}, \sigma_{12}\right)^{T} \\
& \boldsymbol{\varepsilon}=\left(\varepsilon_{11}, \varepsilon_{22}, 2 \varepsilon_{12}\right)^{T}
\end{aligned}
$$

Defining the strain operator $\mathbf{S}$ as

$$
\mathbf{S}=\left(\begin{array}{cc}
\partial_{x} & 0 \\
0 & \partial_{y} \\
\partial_{y} & \partial_{x}
\end{array}\right)
$$

the balance of momentum and constitutive equations can be written as

$$
\frac{\partial \mathbf{v}}{\partial t}=\frac{1}{\rho} \mathbf{S}^{T} \boldsymbol{\sigma}
$$

and

$$
\frac{\partial \boldsymbol{\sigma}}{\partial t}=\mathbf{D}^{e} \mathbf{S v}-\mathbf{D}^{e} \frac{\partial \boldsymbol{\varepsilon}^{v p}}{\partial t}
$$

Therefore, the system above is a system of first order hyperbolic equations, where the nonlinearity affects only the source terms $\mathbf{D}^{e} \frac{\partial \boldsymbol{\varepsilon}^{v p}}{\partial t}$.

The momentum and constitutive Equations [11] and [12] can be written as

$$
\frac{\partial}{\partial t}\left[\begin{array}{c}
\sigma_{11} \\
\sigma_{22} \\
\sigma_{12} \\
v_{1} \\
v_{2}
\end{array}\right]-\frac{\partial}{\partial x}\left[\begin{array}{c}
D_{11} v_{1} \\
D_{12} v_{1} \\
D_{33} v_{2} \\
\frac{\sigma_{11}}{\rho} \\
\frac{\sigma_{12}}{\rho}
\end{array}\right]-\frac{\partial}{\partial y}\left[\begin{array}{c}
D_{12} v_{2} \\
D_{22} v_{2} \\
D_{33} v_{1} \\
\frac{\sigma_{12}}{\rho} \\
\frac{\sigma_{22}}{\rho}
\end{array}\right]=\left[\begin{array}{c}
-D_{11} \frac{\partial \varepsilon_{11}^{v p}}{\partial t}-D_{12} \frac{\partial \varepsilon_{22}^{v p}}{\partial t} \\
-D_{12} \frac{\partial \varepsilon_{11}^{v p}}{\partial t}-D_{22} \frac{\partial \varepsilon_{22}^{v p}}{\partial t} \\
-D_{33} \frac{\partial \varepsilon_{12}^{v p}}{\partial t} \\
0 \\
0
\end{array}\right]
$$

being $D_{\mathrm{ij}}$ the components of the elastic matrix $\mathbf{D}^{e}$. 
The system [13] can be alternatively written as

$$
\frac{\partial \mathbf{U}}{\partial t}+\frac{\partial \mathbf{F}_{x}}{\partial x}+\frac{\partial \mathbf{F}_{y}}{\partial y}=\mathbf{S}
$$

where

$$
\begin{aligned}
\mathbf{U}^{T} & =\left(\begin{array}{lllll}
\sigma_{11} & \sigma_{22} & \sigma_{12} & v_{1} & v_{2}
\end{array}\right) \\
\mathbf{F}_{x}^{T} & =\left(\begin{array}{llllll}
-D_{11} v_{1} & -D_{12} v_{1} & -D_{33} v_{2} & -\frac{\sigma_{11}}{\rho} & -\frac{\sigma_{12}}{\rho}
\end{array}\right) \\
\mathbf{F}_{y}^{T} & =\left(\begin{array}{llllll}
-D_{12} v_{2} & -D_{22} v_{2} & -D_{33} v_{1} & -\frac{\sigma_{12}}{\rho} & -\frac{\sigma_{22}}{\rho}
\end{array}\right) \\
\mathbf{S}^{T} & =\left(\begin{array}{llllll}
-D_{11} \frac{\partial \varepsilon_{11}^{v p}}{\partial t}-D_{12} \frac{\partial \varepsilon_{22}^{v p}}{\partial t} & -D_{12} \frac{\partial \varepsilon_{11}^{v p}}{\partial t}-D_{22} \frac{\partial \varepsilon_{22}^{v p}}{\partial t} & -D_{33} \frac{\partial \varepsilon_{12}^{v p}}{\partial t} & 0 & 0
\end{array}\right)
\end{aligned}
$$

or, in a more compact manner

$$
\frac{\partial \mathbf{U}}{\partial t}+\nabla \mathbf{F}=\mathbf{S}
$$

being $\mathbf{U}$ the unknown vector, $\mathbf{F}$ the advective flux tensor and $\mathbf{S}$ the source vector.

It is interesting to note that in $1 \mathrm{D}$, the system [15] can be written as

$$
\frac{\partial}{\partial t}\left(\begin{array}{l}
\sigma \\
v
\end{array}\right)+\left(\begin{array}{cc}
0 & -E \\
-\frac{1}{\rho} & 0
\end{array}\right) \frac{\partial}{\partial x}\left(\begin{array}{l}
\sigma \\
v
\end{array}\right)=\left(\begin{array}{c}
-E \frac{\partial \varepsilon^{v p}}{\partial t} \\
0
\end{array}\right)
$$

In this case, the velocity of wave propagation is given by the eigenvalues of the matrix $\left(\begin{array}{cc}0 & -E \\ -1 / \rho & 0\end{array}\right): c= \pm \sqrt{E / \rho}$, which do not depend on the stress, strain or material history, and always remain real.

\section{Numerical discretization}

In the presence of discontinuities, such as shock waves, the SPH method presents some numerical problems, such as numerical dispersion and diffusion close to the discontinuities. To overcome these difficulties, the authors propose here an alternative method which consists of applying first the time discretization by means of a Taylor series expansion in two steps and thereafter the space discretization using the corrected SPH method with two different sets of particles. 


\subsection{Two-steps time discretization}

Time discretization of equation [15] is carried out by means of a Taylor series expansion in time of $\mathbf{U}$ up to second order accuracy:

$$
\mathbf{U}^{n+1}=\mathbf{U}^{n}+\left.\Delta t \frac{\partial \mathbf{U}}{\partial t}\right|^{n}+\left.\frac{\Delta t^{2}}{2} \frac{\partial^{2} \mathbf{U}}{\partial t^{2}}\right|^{n}+\mathrm{O}(\Delta t)^{3}
$$

The first order time derivative of the unknowns can be calculated using equation [15]

$$
\left.\frac{\partial \mathbf{U}}{\partial t}\right|^{n}=(\mathbf{S}-\nabla \mathbf{F})^{n}
$$

The second order derivative with respect to time is given by

$$
\left.\frac{\partial^{2} \mathbf{U}}{\partial t^{2}}\right|^{n}=\frac{\partial}{\partial t}(\mathbf{S}-\operatorname{div} \mathbf{F})^{n}=\left(\frac{\partial \mathbf{S}}{\partial t}\right)^{n}-\nabla\left(\frac{\partial \mathbf{F}}{\partial t}\right)^{n}
$$

First step. In order to obtain the time derivatives of fluxes and sources at time $t^{\text {n }}$, the values of the unknowns at an intermediate time $t^{\mathrm{n}+1 / 2}$ will be obtained first

$$
\mathbf{U}^{n+1 / 2}=\mathbf{U}^{n}+\frac{\Delta t}{2}(\mathbf{S}-\nabla \mathbf{F})^{n}
$$

Using the computed value of $\mathbf{U}^{\mathrm{n}+1 / 2}$, fluxes and sources can be evaluated:

$$
\left(\frac{\partial \mathbf{F}}{\partial t}\right)^{n}=\frac{2}{\Delta t}\left(\mathbf{F}^{n+1 / 2}-\mathbf{F}^{n}\right) \text { and }\left(\frac{\partial \mathbf{S}}{\partial t}\right)^{n}=\frac{2}{\Delta t}\left(\mathbf{S}^{n+1 / 2}-\mathbf{S}^{n}\right)
$$

which can now be substituted in equation [18], resulting on

$$
\left.\frac{\partial^{2} \mathbf{U}}{\partial t^{2}}\right|^{n}=\frac{2}{\Delta t}\left(\mathbf{S}^{n+1 / 2}-\mathbf{S}^{n}-\nabla\left(\mathbf{F}^{n+1 / 2}-\mathbf{F}^{n}\right)\right)
$$

Second step. Substituting now the expressions obtained for the first and second order time derivatives, [17] and [21], in the Taylor series expansion [16], the following expression is obtained for the values of the unknowns at time $t^{\mathrm{n}+1}$

$$
\mathbf{U}^{n+1}=\mathbf{U}^{n}+\Delta t(\mathbf{S}-\nabla \mathbf{F})^{n+1 / 2}
$$

\subsection{SPH spatial discretization}

In this section, a brief summary of the basic SPH method and its corrected form, used to improve the accuracy of the results, is presented. 


\subsubsection{Discrete approximation of functions and derivatives}

The kernel approximation of an arbitrary function $f$ is defined by:

$$
\langle f(x)\rangle=\int_{\Omega} f\left(x^{\prime}\right) W\left(x-x^{\prime}, h\right) d x^{\prime}
$$

where the brackets denote a kernel approximation, $x$ and $x$ ' are scalars or vectors, $W(x-x, h)$ is the kernel function and $h$ is a measure of the size of the kernel support. It is clear that the kernel approximation $\langle f(x)\rangle$ converges to the exact function $f(x)$ if the kernel function $W(x, h)$ tends to the Dirac function $\delta(x)$.

The accuracy of the SPH method depends on the properties of the kernel $W(x, h)$. It must be an even function and has to fulfil the following conditions:

(i) $W(x, h) \geq 0$

(ii) $\int_{\Omega} W\left(x-x^{\prime}, h\right) d x=1$

(iii) $W\left(x-x^{\prime}, h\right)=0$ when $\left|x-x^{\prime}\right|>\kappa h$; being $\kappa$ a constant positive parameter

(iv) $\lim _{h \rightarrow 0} \int_{\Omega} W\left(x-x^{\prime}, h\right) d x^{\prime}=\delta\left(x-x^{\prime}\right)$

The SPH approximation can be formulated in terms of the spatial coordinates (Eulerian kernel) or the material coordinates (Lagrangian kernel). It has been shown by Belytschko and co-workers (Belytschko et al., 2000) that the Lagrangian kernel eliminates the tensile instability, and therefore it will be used here. Thus the Lagrangian kernel is expressed in terms of the material coordinates as $W\left(X-X^{\prime}, h_{0}\right)$, being $h_{0}$ the size of the kernel support which will remain constant during the course of the simulation.

In this work the B-spline function based on the cubic spline functions, previously used by Monaghan and Lattanzio (Monaghan et al., 1985), has been chosen as the kernel function:

$$
W(\xi)=C\left\{\begin{array}{lr}
\left(\frac{2}{3}-\xi^{2}+\frac{1}{2} \xi^{3}\right) & 0 \leq \xi \leq 1 \\
\frac{1}{6}(2-\xi)^{3} & 1 \leq \xi \leq 2 \\
0 & \xi \geq 2
\end{array}\right.
$$


being $\xi=\frac{\left|X-X^{\mid}\right|}{h_{0}}=\frac{r}{h_{0}}$; the scaling factor $C$ is given by $\frac{1}{h_{0}}, \frac{15}{7 \pi h_{0}{ }^{2}}$ and $\frac{3}{2 \pi h_{0}{ }^{3}}$ in $1 \mathrm{D}$, $2 \mathrm{D}$ and $3 \mathrm{D}$ respectively.

In the SPH method, a continuum is represented by a set of particles, thus it is necessary to approximate the integral [23] in a discrete manner:

$$
f_{I}=\sum_{J=1}^{N} f_{J} W_{I J} \frac{m_{J}}{\rho_{J}}
$$

where the summation subscript $J$ denotes a particle label and runs over all particles, $N$, inside the domain, such that $\left|X_{I}-X_{J}\right| \leq \kappa h_{0} . W_{I J}=W\left(X_{I}-X_{J}, h_{0}\right)$ denotes the value of the kernel, centred at node $I$, at position $J ; m_{J}$ and $\rho_{J}$ are the mass and density associated to particle $J$ and $f\left(X_{J}\right)=f_{J}$.

The SPH integral representation of the derivative of a function $f(X)$ is given by

$$
\langle\nabla f(X)\rangle=-\int_{\Omega} f\left(X^{\prime}\right) \nabla W\left(X-X^{\prime}, h_{0}\right) d X^{\prime}
$$

where $\nabla W$ is the derivative of $W$ with respect to $X^{\prime}$.

Following the same procedure as above and taking into account that $\sum_{J=1}^{N} f_{I} \nabla W_{I J} \frac{m_{J}}{\rho_{J}}=0$, the discrete form of [26] is obtained:

$$
\nabla f_{I}=\sum_{J=1}^{N} \frac{m_{J}}{\rho_{J}}\left(f_{J}-f_{I}\right) \nabla W_{I J}
$$

where $\nabla f_{I}=\left\langle\nabla f\left(X_{I}\right)\right\rangle ; \nabla W_{I J}=\frac{W^{\prime}}{h_{0}} \frac{X_{I J}}{r_{I J}} ; X_{L J}=X_{I}-X_{J} ; r_{I J}=\left|X_{I J}\right|$

Since, the completeness is given by the order of the polynomial which can be represented exactly, equation [27] satisfies only the zeroth order derivative completeness condition.

It is well known that for boundary particles, the consistency conditions are not satisfied when using approximations [25] and [27]. Many works have been proposed in the past to improve the particle consistency of the SPH method (Belytschko et al., 2000) (Belytschko et al., 1996) (Bonet et al., 2000) (Johnson et al., 1996) (Krongauz et al., 1997) (Rabczuk et al., 2004) (Randles et al., 2000) (Xiao et al., 2005]. Here the correction of the approximating function and its derivatives are given in order to satisfy the zeroth and the first order completeness respectively. 
- Corrected form of the approximating function

To fulfill the zeroth order completeness of the approximation in the whole domain, the corrected particle approximation for any function $f$ must be used:

$$
f_{I}=\sum_{J=1}^{N} f_{J} \tilde{W}_{I J} \Omega_{J}=\frac{\sum_{J=1}^{N} f_{J} W_{I J} \Omega_{J}}{\sum_{J=1}^{N} W_{I J} \Omega_{J}}
$$

being $\widetilde{W}_{I J}=\frac{W_{I J}}{\sum_{J=1}^{N} W_{I J} \Omega_{J}}$ the corrected kernel, and $\Omega_{J}=\frac{m_{J}}{\rho_{J}}$.

In the inner domain, the denominator of above equation is equal to 1 and the original SPH and its corrected form are identical.

- Corrected form of the derivatives of the approximating function

In order to fulfil the first order completeness, the corrected form of the derivative of the approximating function must be used:

$$
\nabla f_{I}=\left(\sum_{J=1}^{N} f_{I J} \nabla W_{I J} \Omega_{J}\right) \mathbf{C}_{I}=\sum_{J=1}^{N} f_{I J} \widetilde{\nabla} W_{I J} \Omega_{J}
$$

being $f_{I J}=f_{I}-f_{J} ; X_{I J}=X_{I}-X_{J} ; \Omega_{J}=\frac{m_{J}}{\rho_{J}}$

where $\quad \mathbf{C}_{I}=\left(\sum_{J=1}^{N} X_{I J} \otimes \nabla W_{I J} \Omega_{J}\right)^{-1}$

and being $\widetilde{\nabla} W_{I J}=\mathbf{C}_{I} \nabla W_{I J}$ the corrected derivative of the kernel.

The correction proposed here is similar to the SPH normalization developed by Randles and Libersky for an Eulerian kernel (Randles et al., 1997).

\subsubsection{Discretized equations using the proposed method: Taylor-SPH}

As it was explained before, in this work a new SPH formulation is proposed (Taylor-SPH). The numerical model is formulated in terms of stress and velocity instead of displacement, as it was traditionally done with $\mathrm{SPH}$, and a corrected Lagrangian kernel is used for the spatial discretization. The time discretization is carried out in two steps by means of a Taylor series expansion. As a result of the 
overall formulation of the method, this new SPH formulation avoids numerical instabilities and minimizes numerical dispersion and diffusion.

To perform the proposed time discretization, it is necessary the use of an auxiliary set of particles: the "virtual" particles. This auxiliary set of particles plays a similar role as the Gauss points do in the Taylor-Galerkin algorithm used in FEM, allowing the calculation of the unknowns at an intermediate time $t^{\mathrm{n}+1 / 2}$. Otherwise, the time discretization could not be carried out in two steps. These "virtual" particles will be interspersed among the "real" particles in a similar manner it was done in stresspoint integration methods. Figure 1 shows the arrangement of "real" and "virtual" particles in one and two dimensions.

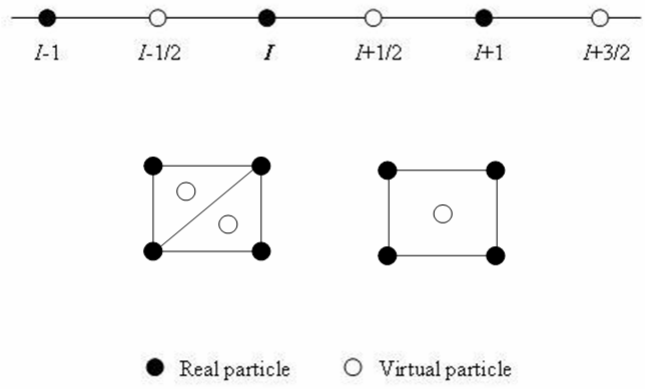

Figure 1. "Real" and "virtual" particles

Thus, time discretization of model equations is carried out in two steps:

- In the first step, the values of the field variables at time $t^{\mathrm{n}+1 / 2}$ are computed by means of equation [19] at the positions of the $N v$ "virtual" particles.

- In the second step, the values of the field variables at time $t^{\mathrm{n}+1}$ are computed, using equation [22], at the positions of the $\mathrm{Nr}$ "real" particles.

First Step. Applying the corrected SPH spatial discretization to the first step of time discretization, [19], we obtain:

$$
\langle\mathbf{U}\rangle_{V P}^{n+1 / 2}=\langle\mathbf{U}\rangle_{V P}^{n}+\left\langle\frac{\Delta t}{2}\right\rangle\left[\langle\mathbf{S}\rangle_{V P}^{n}-\langle\nabla \mathbf{F}\rangle_{V P}^{n}\right]
$$

The subscript $V P$ indicates that the values of $\mathbf{U}, \mathbf{S}$ and $\nabla \mathbf{F}$ are computed at the positions of the "virtual" particles.

Using the corrected form for the approximation of functions and derivatives given by Equations [28] and [29], we obtain the values of the variable $\mathbf{U}$ at $t^{\mathrm{n}+1 / 2}$ : 


$$
\mathbf{U}_{V P}^{n+1 / 2}=\mathbf{U}_{V P}^{n}+\frac{\Delta t}{2}\left[\sum_{J=1}^{N r} \frac{m_{J}}{\rho_{J}} \mathbf{S}_{J}^{n} \widetilde{W}_{I J}-\sum_{J=1}^{N r} \frac{m_{J}}{\rho_{J}} \mathbf{F}_{J}^{n} \tilde{\nabla} W_{L J}\right]
$$

being $J$ the "real" particles, such that $\left|X_{J}-X_{V P}\right| \leq 2 h_{0}$.

Second Step. Applying the corrected SPH spatial discretization to equation [22], it is obtained

$$
\langle\mathbf{U}\rangle_{R P}^{n+1}=\langle\mathbf{U}\rangle_{R P}^{n}+\langle\Delta t\rangle\left\langle\langle\mathbf{S}\rangle_{R P}^{n+1 / 2}-\langle\nabla \mathbf{F}\rangle_{R P}^{n+1 / 2}\right\rfloor
$$

The subscript $R P$ indicates that the values of $\mathbf{U}, \mathbf{S}$ and $\nabla \mathbf{F}$ are computed at the positions of the "real" particles.

Using expressions [28] and [29], the values of the variable $\mathbf{U}$ at $t^{\mathrm{n}+1}$ are given by:

$$
\mathbf{U}_{R P}^{n+1}=\mathbf{U}_{R P}^{n}+\Delta t\left[\sum_{J=1}^{N_{v}} \frac{m_{J}}{\rho_{J}} \mathbf{S}_{J}^{n+1 / 2} \widetilde{W}_{I J}-\sum_{J=1}^{N_{v}} \frac{m_{J}}{\rho_{J}} \mathbf{F}_{J}^{n+1 / 2} \tilde{\nabla} W_{I J}\right]
$$

where $J$ are the "virtual" particles, such that $\left|X_{J}-X_{R P}\right| \leq 2 h_{0}$.

The particle masses and volumes needed to calculate [32] and [34] are obtained via Voronoi diagram. In the 2D procedure, we use a structured quadrilateral particle arrangement. As it is shown in Figure 2: 4 "real" particles are arranged so they form a square and a "virtual" particle for the calculations at $t^{n+1 / 2}$ is placed in the centroid of the square.

In this particular case, computation of particle volumes is especially easy. The square is then subdivided into 4 other squares for computation of the volumes, and once the coordinates of the "real" and "virtual" particles are known, quadrilateral volumes can be easily computed as it is shown in Figure 2.

It is important to note here that in the first step, [32], only volumes of "real" particles are considered, while in the second step, [34], only volumes of "virtual" particles are taken into account. Note that particle masses and volumes need to be computed only once for a Lagrangian kernel.

It is also important to mention that the method presented here does not require any special treatment of the boundary conditions.

In the following, the new SPH formulation using the two-steps time discretization scheme presented here will be referred to as TSPH, which stands for Taylor-SPH. 


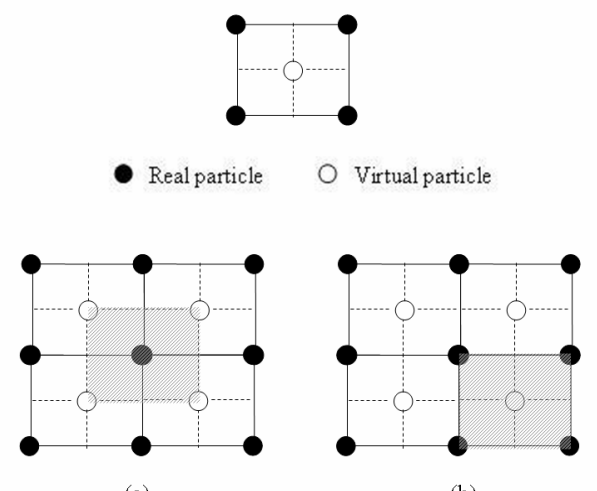

(a)

(b)

Figure 2. "Real" and "virtual" particles arrangement in a structured quadrilateral configuration: (a) Quadrilateral volume of "real” particles; (b) Quadrilateral volume of "virtual" particles

\subsection{FEM discretization: Taylor-Galerkin}

In order to compare the results obtained using TSPH with those obtained with the Taylor-Galerkin algorithm using FEM, a brief summary of the Taylor-Galerkin algorithm will be given.

The method consists of applying first the time discretization explained in Section 3.1. After that, the standard Galerkin method is applied to equation [22], resulting on the following discrete system of equations:

$$
\mathbf{M} \Delta \mathbf{U}=\Delta t\left[\int_{\Omega} \mathbf{N}^{T} \mathbf{S}^{n+1 / 2} d \Omega+\int_{\Omega} \mathbf{F}^{n+1 / 2} \nabla \mathbf{N} d \Omega-\int_{\Gamma_{N}} \mathbf{N}^{T}\left(\mathbf{F}^{n+1 / 2} \cdot \mathbf{n}\right) d \Gamma\right]
$$

where $\mathbf{N}$ is the shape function, $\mathbf{M}$ is the mass matrix and $\mathbf{n}$ is the normal to the boundary $\Gamma_{\mathrm{N}}$.

The discrete system above is of the form

$$
\mathbf{M} \mathbf{x}=\mathbf{f}
$$

and can be efficiently solved using, for instance, the Jacobi iteration scheme. 


\section{Numerical examples}

Numerical modelling of propagation of waves that present discontinuities, such as shock waves, presents some major difficulties: numerical dispersion and diffusion problems. The amplitude of the wave decreases due to numerical damping and oscillations appear close to the discontinuities due to numerical dispersion of the scheme.

The authors have addressed this problem in previous works using the Finite Element Method, finding out that these difficulties are more important when using formulations in terms of displacements and less important when using the mixed formulation in terms of stress and velocity (Mabssout et al., 2003a) (Mabssout et al., 2003b) (Mabssout et al., 2006).

It will be presented here some examples of propagation of discontinuous waves in elasto-viscoplastic media in order to show the performance of the proposed TSPH method in comparison with other methods used by the authors within the frame of the Finite Element Method.

\subsection{Propagation of a shock wave on a $1 \mathrm{D}$ bar}

In this section it will be solved the problem of a shock wave propagating in both, an elastic and viscoplastic 1D bar, using the proposed TSPH method. The results will be compared with those obtained in previous works with FEM using a classical displacement formulation with the Newmark scheme and using a mixed formulation, in terms of stress and velocity, with the Taylor-Galerkin algorithm.

The Newmark family of methods can be written in terms of displacement as follows (Newmark, 1959):

$$
\begin{aligned}
& u^{n+1}=u^{n}+\Delta t \dot{u}^{n}+\frac{\Delta t^{2}}{2}\left[(1-\beta) \ddot{u}^{n}+\beta \ddot{u}^{n+1}\right] \\
& \dot{u}^{n+1}=\dot{u}^{n}+\Delta t\left[(1-\gamma) \ddot{u}^{n}+\gamma \ddot{u}^{n+1}\right]
\end{aligned}
$$

The Newmark family contains as special cases many well-known and wide used methods. The $\beta$ and $\gamma$ parameters determine the stability of the particular method under consideration. When dealing with dynamic problems and large-scale systems a solution by an explicit Newmark scheme $(\beta=0, \gamma=0.5)$ may require many thousands of time-steps to cover a specified time interval. Thus, for dynamic applications such as shock waves, it is often more efficient to deal with the average acceleration method $(\beta=0.5, \gamma=0.5)$ which is unconditionally stable (Hughes, 1987) (Zienkiewicz et al., 2000). Therefore, in the following, the average acceleration method will be used instead of the explicit Newmark scheme which is not felt to be economically competitive. 


\subsubsection{Elastic case}

The problem consists of a bar of length $L=1 \mathrm{~m}$ with a unit section, which has been spatially discretized using 100 particles (100 "real" particles and 99 "virtual" particles for TSPH).

The boundary conditions are given en terms of velocities:

(i) On $x=0$ (left boundary): $v(0, t)=\left\{\begin{array}{c}1 \mathrm{~m} / \mathrm{s} \quad \text { for } \quad t \leq t_{f}=2.5 \mathrm{~ms} \\ 0 \quad \text { for } \quad t>t_{f}=2.5 \mathrm{~ms}\end{array}\right.$

(ii) On $x=L$ (right boundary): $v(L, t)=0$

The initial conditions are: $v(x, 0)=0 ; \sigma(x, 0)=0$

Material properties are: $\rho=2000 \mathrm{~kg} / \mathrm{m}^{3} ; E=810^{7} \mathrm{~Pa}$.

There is an analytical solution available for this elastic problem: the incoming wave will propagate towards the right boundary without any distortion and keeping its initial amplitude of $\sigma_{0}=v_{0} \sqrt{\rho E}=4.10^{5} \mathrm{~Pa}$ and $v_{0}=1 \mathrm{~m} / \mathrm{s}$. It will reflect at the fixed end $(L=1 \mathrm{~m})$ and the amplitude of $\sigma$ at this point will be doubled to a value of $8.10^{5} \mathrm{~Pa}$, while the velocity of the wave after reflexion will propagate along the bar with $v_{0}=-1 \mathrm{~m} / \mathrm{s}$.

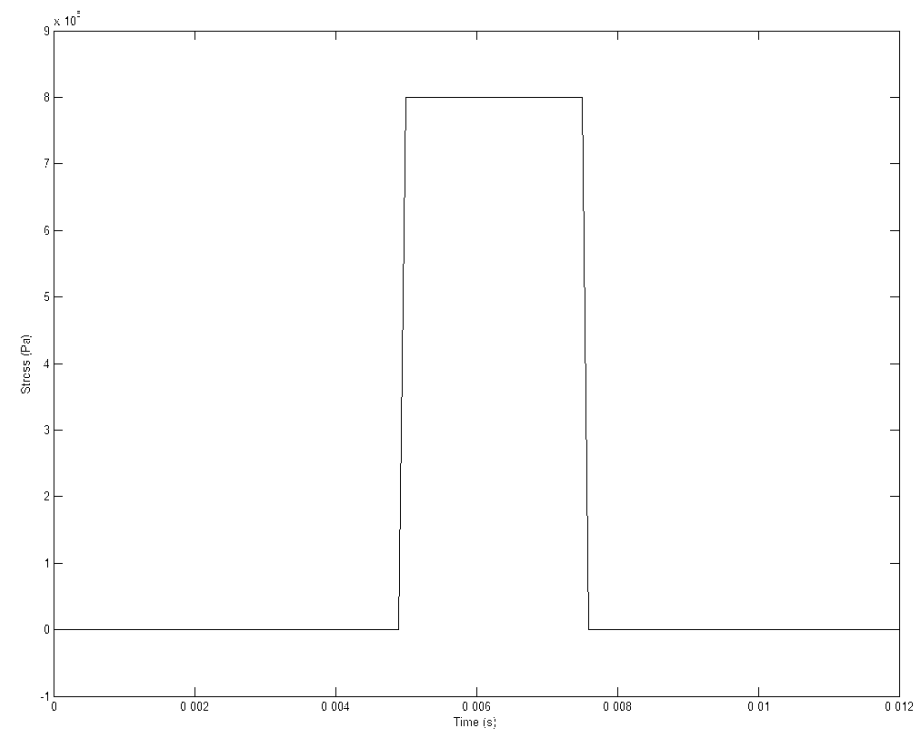

Figure 3. Stress $\sigma(x=1 \mathrm{~m} ; t)$ in the elastic case with TSPH 
296 European Journal of Computational Mechanics. Volume 20 - No. 5-6/2011

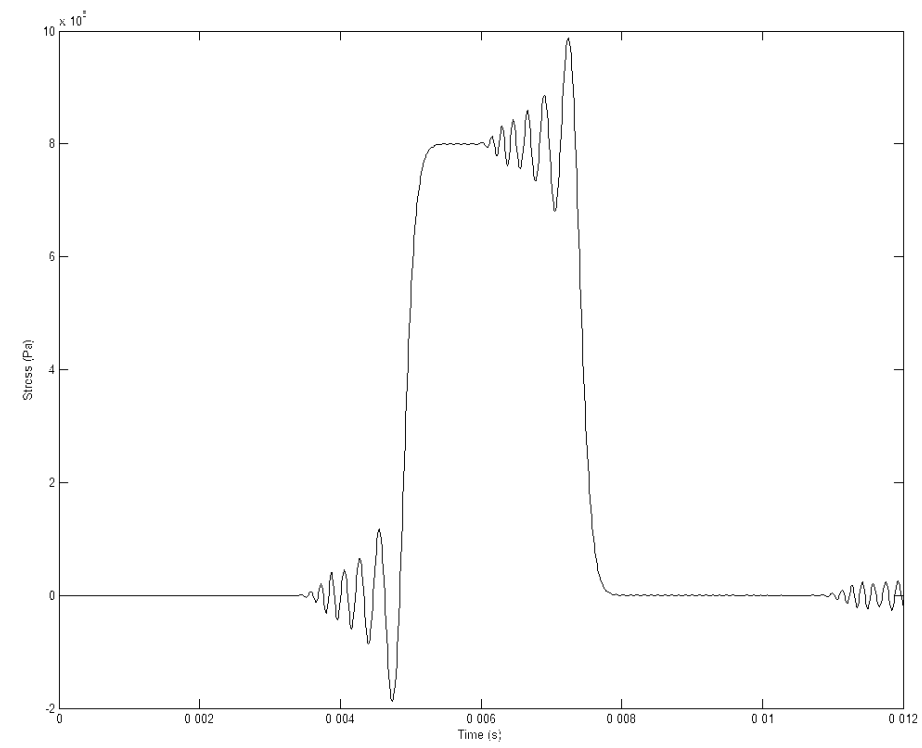

Figure 4. Stress $\sigma(x=1 \mathrm{~m} ; t)$ in the elastic case with FEM using displacement formulation with Newmark $(\beta=0.5, \gamma=0.5)$

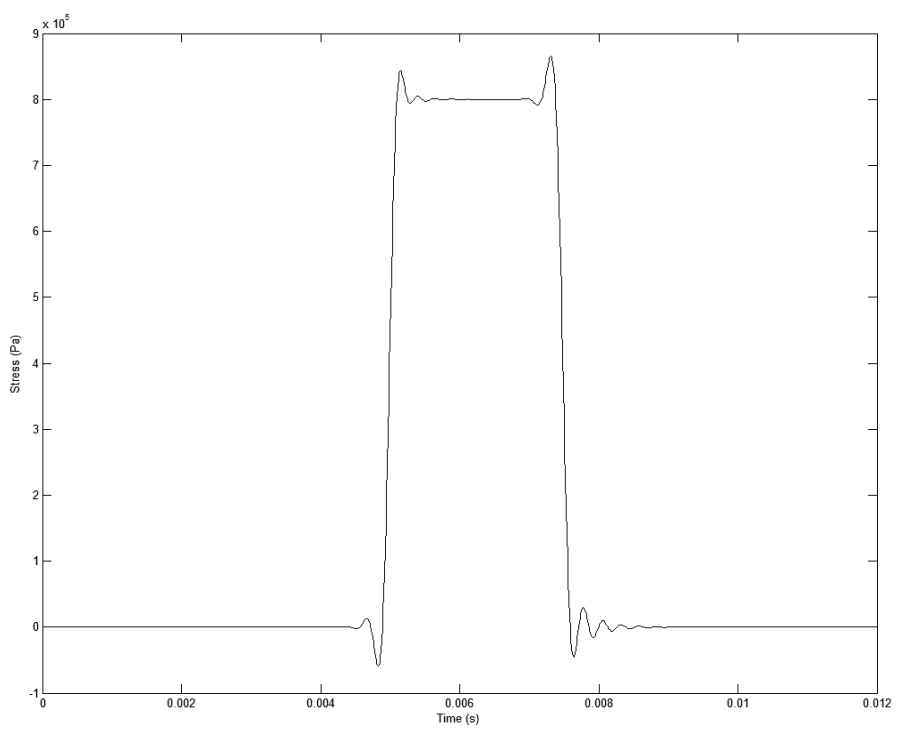

Figure 5. Stress $\sigma(x=1 \mathrm{~m} ; t)$ in the elastic case with FEM using mixed formulation with Taylor-Galerkin 
Figure 3 shows the stress $\sigma(x=1, t)$, after reflection of the wave, obtained with the proposed TSPH method. The time-step used in the calculation is $\Delta \mathrm{t}=510^{-5} \mathrm{~s}$. It can be observed that no oscillations appear in the front of the wave and the results are in complete agreement with the analytical solution.

However, oscillations appear in the front of the wave when using FEM with the average acceleration Newmark scheme (Figure 4) and Taylor-Galerkin algorithm (Figure 5).

Figure 6 shows the velocity at $x=0.5 \mathrm{~m}$. It can be observed that both, the incoming and the reflected waves are in very good agreement with the analytical solution when using the proposed TSPH. However, in the solution obtained using the Taylor-Galerkin algorithm oscillations appear close to the discontinuities (Figure 7).

These results show the good performance of the proposed TSPH method when solving problems of propagation of discontinuous waves in an elastic bar in comparison with other methods proposed in the past. In addition to that, the timesteps required to get good accuracy with FEM using Taylor-Galerkin $\left(\Delta t=10^{-5} \mathrm{~s}\right)$ and the average acceleration Newmark scheme $\left(\Delta \mathrm{t}=810^{-6}\right)$ are smaller than the time-step required by TSPH $\left(\Delta \mathrm{t}=510^{-5} \mathrm{~s}\right)$, and therefore the efficiency of the proposed scheme is higher.

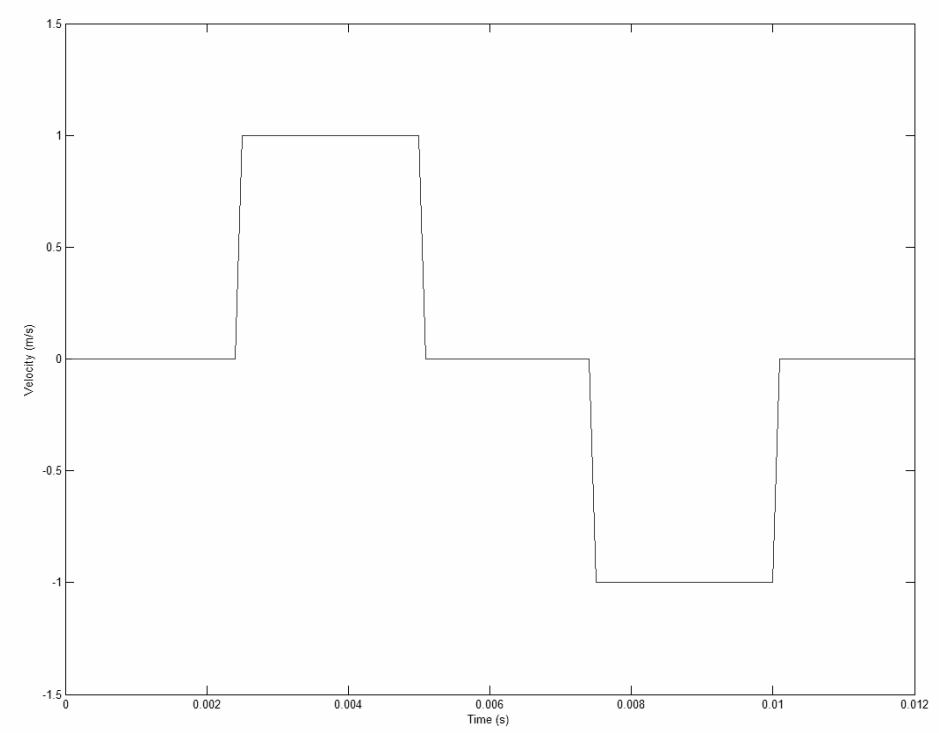

Figure 6. Velocity $v(x=0.5 \mathrm{~m} ; t)$ in the elastic case with TSPH 


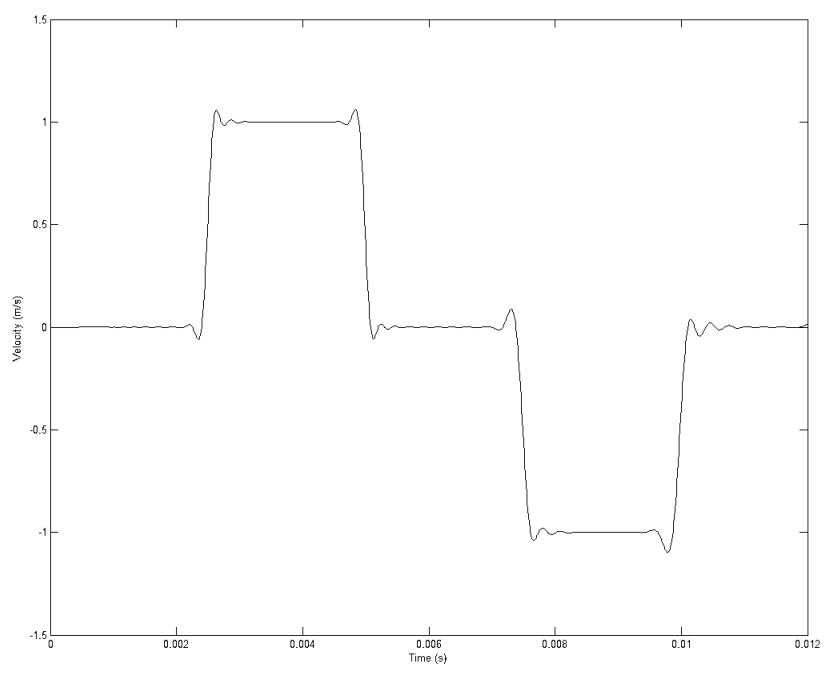

Figure 7. Velocity $v(x=0.5 \mathrm{~m} ; t)$ in the elastic case with FEM using mixed formulation with Taylor-Galerkin

\subsubsection{Viscoplastic case}

It will be analyzed now the propagation of a shock wave in a viscoplastic softening bar. The Perzyna's viscoplastic model is considered (Perzyna, 1966).

The problem consists of a bar of length $L=1 \mathrm{~m}$ with a unit section, which has been spatially discretized using 100 particles (100 "real" particles and 99 "virtual" particles for TSPH). The problem has been analysed under the same initial and boundary conditions as in the elastic case.

In the case of $N=0$, the problem has an analytical solution and the stress at $x=L$ is given by

$$
\sigma(L, t)=\left[2 v_{o} \sqrt{\rho E}-\frac{1}{2} E \gamma\left(t-t_{o}\right)\right] H\left(\frac{t-t_{o}}{t_{r}-t}\right)
$$

where: $t_{o}=L \sqrt{\rho / E} ; \quad t_{r}=t_{o}+t_{f}$ being $t_{f}=2.5 \mathrm{~ms} ; H(t)$ is the Heaviside function:

$$
H\left(\frac{t-t_{0}}{t_{r}-t}\right)=\left\{\begin{array}{lll}
0 & \text { for } & t<t_{0} \\
1 & \text { for } & t_{0}<t<t_{r} \\
0 & \text { for } & t>t_{r}
\end{array}\right.
$$


The material properties are: $\rho=2000 \mathrm{~kg} / \mathrm{m}^{3}, \quad E=810^{7} \mathrm{~Pa}$, yield stress $\bar{\sigma}_{0}=410^{5} \mathrm{~Pa}$ and softening modulus $H=-E / 10$. The model parameters are $N=0$ and $\gamma=1 \mathrm{~s}^{-1}$.

Figure 8 presents the comparison between the analytical solution [38] at $L=1 \mathrm{~m}$ and the result obtained using the proposed TSPH method. It can be observed that the analytical and the numerical solutions are in complete agreement since the numerical solution is free of oscillations and diffusion.

Figure 9 shows a comparison between the analytical solution and the results obtained when using FEM with $N=0$ and $\gamma=1$. It can be observed, that in this case oscillations are present in the front of the wave.

It will be also considered the case in which Perzyna parameters are $N=2$ and $\gamma=2 \mathrm{~s}^{-1}$. In this case, an analytical solution is not available. Figure 10 depicts a comparison between the results obtained with the proposed TSPH and with Taylor-Galerkin for the stress at the fixed end of the bar after reflection. It can be observed that the TSPH method provides much better accuracy for the propagation of shock waves than Taylor-Galerkin.

Finally, it is worth mentioning that the time-steps required to get good accuracy with FEM using Taylor-Galerkin $\left(\Delta \mathrm{t}=810^{-6} \mathrm{~s}\right)$ and the average acceleration Newmark method $\left(\Delta \mathrm{t}=810^{-6} \mathrm{~s}\right)$ is again smaller than the time-step required by TSPH $\left(\Delta \mathrm{t}=510^{-5} \mathrm{~s}\right)$, and therefore the efficiency of the proposed scheme in this case is also higher.

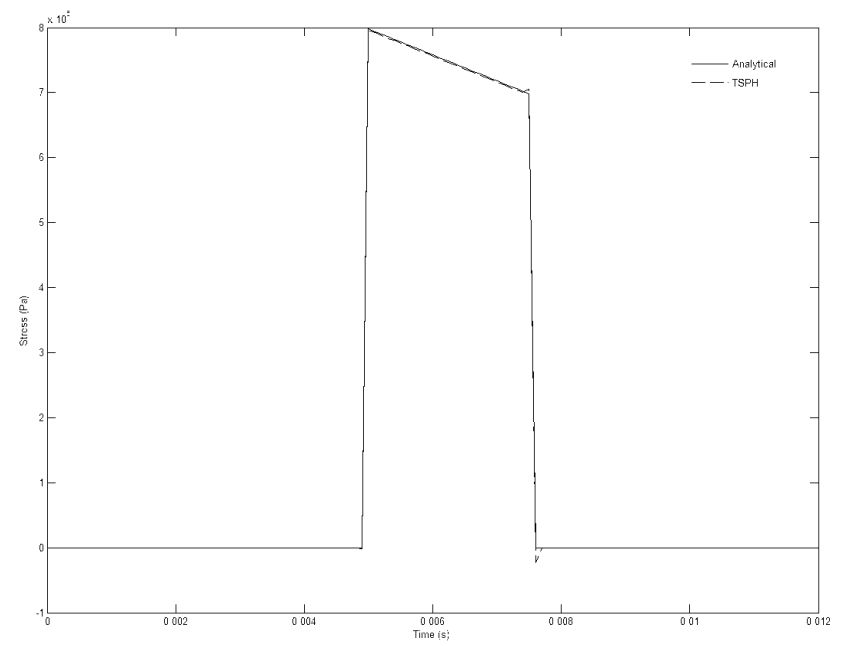

Figure 8. Stress $\sigma(x=1 \mathrm{~m}$; $\mathrm{t})$ in the viscoplastic case for a constant source term $(N$ $=0$ and $\gamma=1)$. Comparison between the analytical solution and the numerical solution obtained with TSPH 
300 European Journal of Computational Mechanics. Volume 20 - No. 5-6/2011

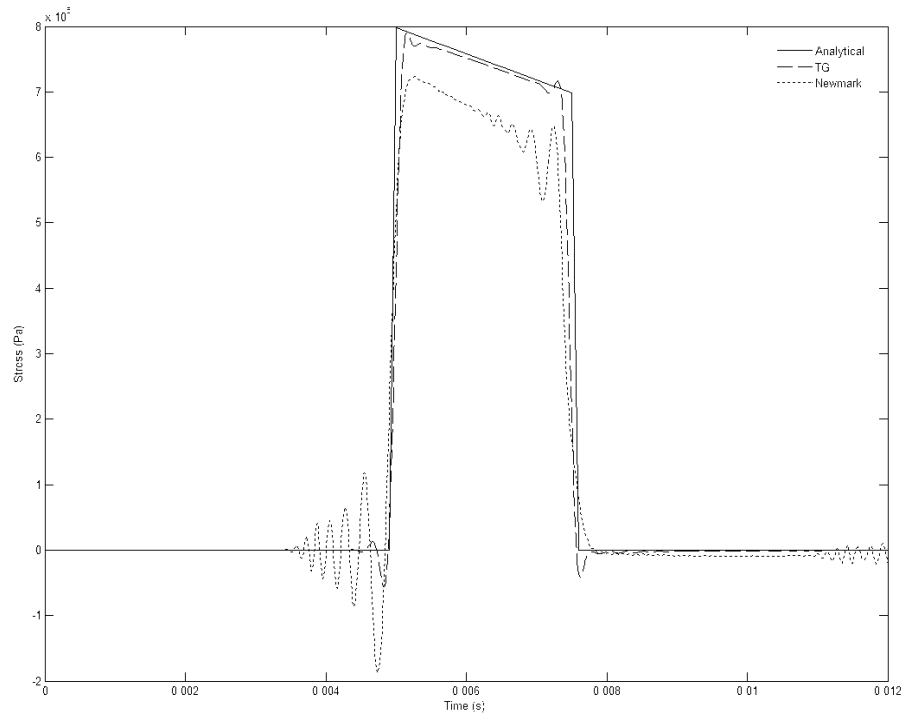

Figure 9. Stress $\sigma(x=1 \mathrm{~m}$; $\mathrm{t})$ in the viscoplastic case for a constant source term ( $N$ $=0$ and $\gamma=1)$. Comparison of the analytical solution with the numerical solution obtained with FEM using Taylor-Galerkin (TG) and the average acceleration Newmark scheme $(\beta=0.5, \gamma=0.5)$

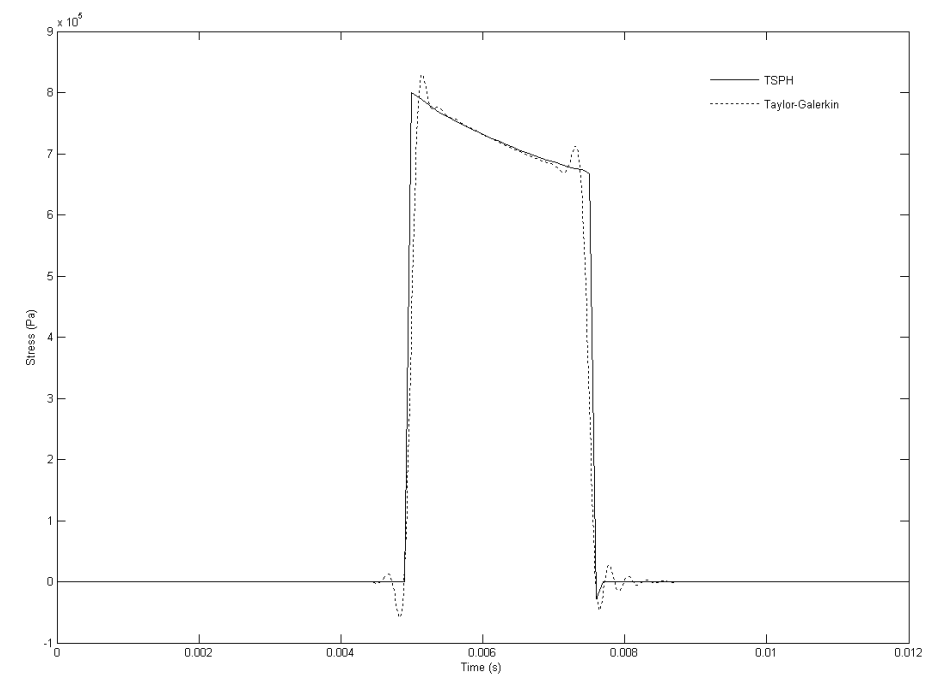

Figure 10. Stress $\sigma(x=1 \mathrm{~m} ; t)$ in the viscoplastic case $(N=2$ and $\gamma=2)$ : TaylorSPH vs Taylor-Galerkin 
With these two examples it has been shown that the proposed TSPH method provides good resolution of shock waves without numerical diffusion and dispersion. Numerical instabilities are not present when using the TSPH method.

In the following example, it will be shown the performance of TSPH in capturing shear bands produced in fast dynamics and shock wave propagation problems.

\subsection{Strain localization in $2 D$ problem}

Simulation of the shear band in a two dimensional specimen subjected to a shock on its upper face (Figure 11) is analyzed here. The spatial domain consists of a square of side $1 \mathrm{~m}$, but for symmetry reasons only one half will be considered in the analysis. It will be compared the shear band obtained with the proposed TSPH method and with the Taylor-Galerkin algorithm used with FEM.

The material behaviour is assumed to obey a viscoplastic Perzyna law with Von Mises yield surface. The parameters used in the computation are:

Density $\rho=2000 \mathrm{~kg} / \mathrm{m}^{3}$, Young modulus $E=810^{7} \mathrm{~Pa}$, Poisson's ratio $v=0.3$, initial yield stress is $\sigma=510^{5} \mathrm{~Pa}$, softening modulus $H=-E / 10$ and model parameters $N=1$ and $\gamma=25$.

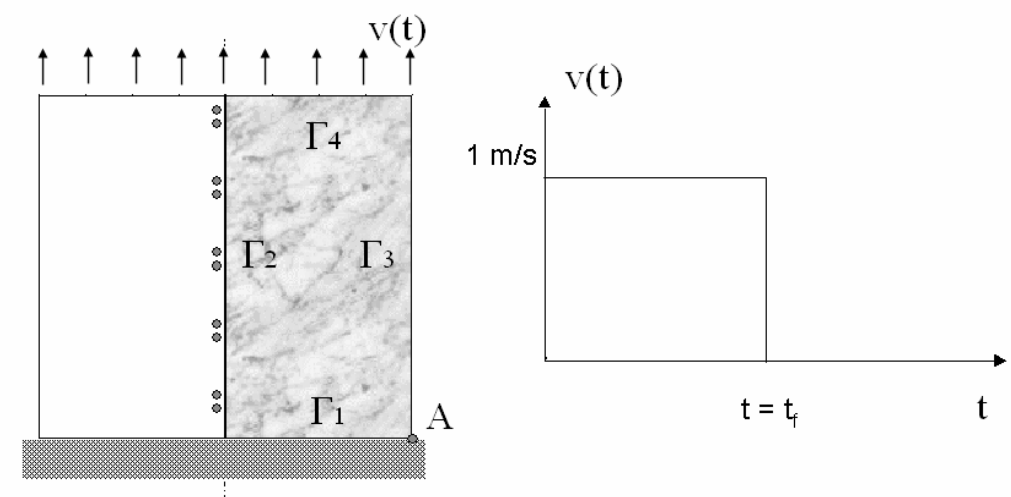

Figure 11. Sketch of the specimen

The domain is discretized using a structured particle arrangement of 861 "real" particles and 800 "virtual" particles (Figure 12), so that every 4 "real" particles form a square with a "virtual" particle placed at its centre. 


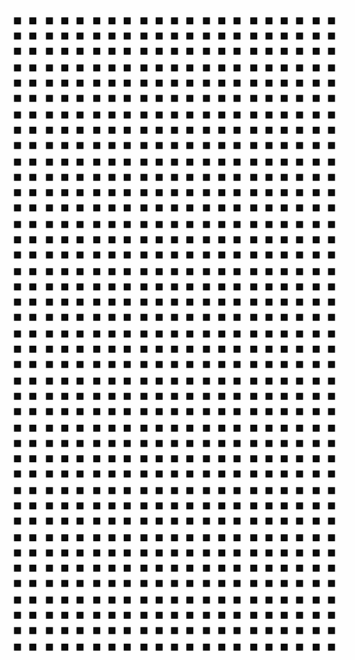

Figure 12. "Real” particles arrangement (21 x 41 "real” particles)

The applied boundary conditions are the following:

(i) On $\Gamma_{1}$ velocity has been set equal zero

(ii) On $\Gamma_{2}$ the symmetry results on $v_{x}=0$ and $\sigma_{x y}=0$

(iii) $\Gamma_{3}$ is a stress free boundary, and therefore $\sigma_{x x}=0$ and $\sigma_{x y}=0$

(iv) Finally, velocity at $\Gamma_{4}$ is: $v_{y}=v(t)$

where $v(t)$ is given by: $v(t)=\left\{\begin{array}{c}1 \mathrm{~m} / \mathrm{s} \quad t \leq t_{f}=8 \mathrm{~ms} \\ 0 \quad t>t_{f}=8 \mathrm{~ms}\end{array}\right.$

Figure 13 shows the viscoplastic strain contours obtained with TSPH. The amplitude of the wave has been chosen such that the stresses do not reach the yield surface until the wave is reflected at the bottom. Hereafter, the stress doubles, the stress path crosses the yield surface, and the strain localizes in the form of a shear band which is incepted at point A (see Figure 11). Inclination of the shear band is close to $45^{\circ}$. Figure 14 depictes the deformed configuration of the "real" particles.

Mesh dependence problems are absent due to the meshfree nature of the TSPH, and numerical instabilities are not present when using the proposed method.

The same problem is solved using the Taylor-Galerkin algorithm with mixed formulation with FEM. A structured mesh of 861 nodes grouped in linear triangular elements T3 has been used and the same boundary conditions as above have been applied (Figure 15). 


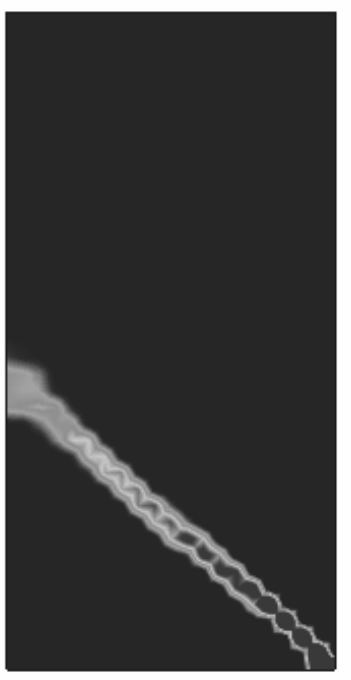

Figure 13. TSPH: Viscoplastic strain (21 $x 41$ "real” particles)

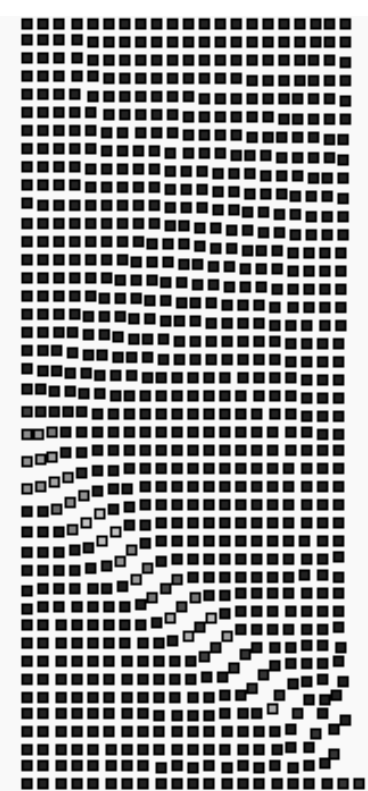

Figure 14. TSPH: Deformed configuration (21 $x 41$ "real” particles) 


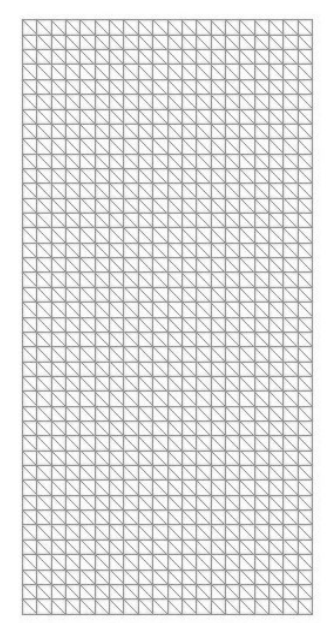

Figure 15. Computational mesh used with FEM (21 x 41 nodes grouped in T3 elements)

Viscoplastic strain and deformed configuration are given in Figures 16 and 17. Comparing these results with those obtained with the proposed TSPH formulation, it can be observed how with the new TSPH method the direction of the shear band is closer to $45^{\circ}$ and the shear band width is smaller.

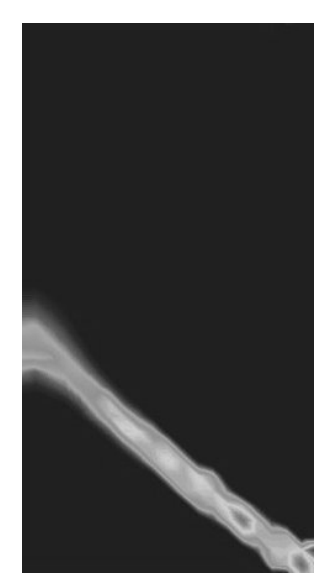

Figure 16. Taylor-Galerkin: Viscoplastic strain (21 $x 41$ nodes) 


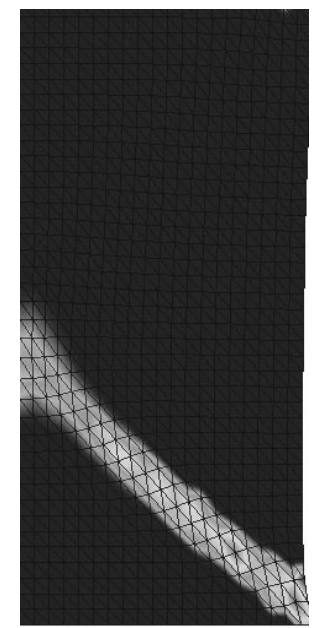

Figure 17. Taylor-Galerkin: Deformed mesh (21 $x 41$ nodes)

The time-step required to get good accuracy with FEM using Taylor-Galerkin is $\Delta \mathrm{t}=210^{-5} \mathrm{~s}$ and the time-step required by TSPH is $\Delta \mathrm{t}=410^{-5} \mathrm{~s}$. Therefore, we can conclude that the efficiency of the Taylor-SPH is higher.

\section{Conclusions}

The Taylor-SPH method applied to shock waves propagating in viscoplastic media has been presented. The method consists of a two-steps time discretization scheme based on a Taylor series expansion of the stress and velocity fields using a corrected SPH. Two different sets of particles have been used for the computations at each time step and a Lagrangian kernel has been used in order to avoid numerical instabilities. The results have been compared with those obtained using FEM.

In the first example, the problem of the propagation of a shock wave in a bar has been analyzed. The results have been compared with those obtained using the Taylor-Galerkin algorithm with mixed formulation within the frame of the Finite Element Method, and it has been demonstrated how the proposed TSPH method provides more accurate solutions, since numerical problems such as diffusion and dispersion are absent.

To asses the performance of the TSPH method in capturing shear bands, a 2D specimen subjected to a shock on its upper face has been considered. It has been shown again how the proposed TSPH performs better in capturing the shear band than the Taylor-Galerkin algorithm used with FEM. Numerical instabilities are not observed when using the TSPH method with a Lagrangian kernel. 
In these examples, the advantages of using TSPH instead of Taylor-Galerkin have been shown. TSPH provides accurate solutions, avoiding numerical dispersion and diffusion as well as mesh dependence problems inherent to most FEM formulations. In addition to that, time-steps needed for calculations are very competitive and the method has been shown to be stable, robust and only a reduced number of particles is required to obtain reasonably accurate results.

\section{Acknowledgements}

The authors would like to express their gratitude to the Agencia Española de Cooperación Internacional for the economic support granted.

\section{References}

Belytschko T., Guo Y., Liu W. K. and Xiao S. P., "A unified stability analysis of meshless particle methods", Int. J. Numer. Meth. Engrg., 48, 2000, p. 1359-1400.

Belytschko T., Krongauz Y., Dolbow Y., Gerlach C., "On the completeness of meshfree particle methods", Int. J. Numer. Methods Eng., 1998, 43, p. 785-819.

Belytschko T., Krongauz Y., Organ D., Fleming M., Krysl P., "Meshless methods: An overview and recent developments", Comput. Methods. Appl. Mech. Engrg., 1996, 139, p. 3-47.

Bonet J., Kulasegaram S., "Correction and stabilization of smooth particle hydrodynamics methods with applications in metal forming simulations", Int. J. Numer. Meth. Engng. 2000, 47, p. 1189-1214.

Caleyron F., Chuzel-Marmot Y., Combescure A., "Modeling of reinforced concrete through SPH-FE coupling and its application to the simulation of a projectile's impact onto a slab", International Journal for Numerical Methods in Biomedical Engineering, 2009, DOI: $10.1002 / \mathrm{cnm} .1341$

Chuzel-Marmot Y., Combescure A., Ortiz R., "Explicit dynamics 'SPH-Finite Element' coupling using the Arlequin method. Simulation of projectile's impacts on concrete slabs", Revue Européenne de Mécanique Numérique, 2008, p. 737-748.

Dyka C. T., Ingel R. P., "An approach for tension instability in Smoothed Particle Hydrodynamics", Computers and Structures, 1995, 57, p. 573-580.

Dyka C. T., Randles P. W. and Ingel R. P., "Stress points for tension instability in SPH”, Int. J. Numer. Meth. Engrg., 1997, 40, p. 2325-2341.

Gingold R.A., Monaghan J.J., "Smoothed particles hydrodynamics: Theory and application to non-spherical stars", Monthly Notices of the Royal Astronomical Society, 1977, 181, p. $375-389$.

Hughes T.J.R., The Finite Element Method: Linear Static and Dynamic Finite Element Analysis, Prentice-Hall, Inc., Engewood Cliffs, New Jersey, 1987. 
Johnson G.R., Beissel S.R., "Normalized smoothing functions for SPH impact computations", Int. J. Numer. Methods Engrg., 1996, 39, p. 2725-2741.

Krenk S., "Energy conservation in Newmark based time integration algorithms", Computer Methods in Applied Mechanics and Engineering, 2006, 195, 44-47: 6110-6124.

Krenk S. and Høgsberg J., "Properties of time integration with first order filter damping", Int. J. Numer. Meth. Engng, 2005, 64 :547-566.

Krongauz Y., Belytschko T., "Consistent pseudo derivatives in meshless methods", Comput. Meth. Appl. Mech. Engng, 1997, 146: p. 371-386.

Laursen T.A., Computational contact and impact Mechanics: fundamentals of modeling interfacial phenomena in nonlinear finite element analysis, Springer, Berlin, 2002.

Li X., Yao D., Lewis RW., "A discontinuous Galerkin Finite Element Method for dynamic and wave propagation problems in non-linear solids and saturated porous media", International Journal for Numerical Methods in Engineering 2003, 57, p. 1775-1800.

Libersky L.D., Petschek A.G., "Smooth particle hydrodynamics with strength of materials, Advances in the Free Lagrange Method", Lecture Notes in Physics, 1991, 395, p. 248-257.

Liu W. K., Jun S., Zhang Y. F., "Reproducing kernel particle methods", Int. J. Numer. Meth. Engng., 1995, 20, 8-9, p. 1081-1106.

Lucy L.B., "A numerical approach to the testing of fusion process", Astronomical Journal, 1977, 82 p. 1013-1024.

Mabssout M., Pastor M., "A Taylor-Galerkin algorithm for shock wave propagation and strain localization failure of viscoplastic continua", Comput. Methods. Appl. Mech. Engrg, 2003a, 192, p. 955-971.

Mabssout M., Pastor M., “A two step Taylor-Galerkin algorithm for shock wave propagation in soils", Int. J. Numer. Analytical Meth. Geomechanics, 2003b, 27, p. 685-704.

Mabssout M., Pastor M., Herreros M.I., Quecedo M., “A Runge-Kutta, Taylor-Galerkin scheme for hyperbolic systems with source terms. Application to shock wave propagation in viscoplastic geomaterials", Int. J. Numer. Analytical Meth. Geomechanics, 2006, vol. $30, \mathrm{n}^{\circ} 13$, p. 1337-1355.

Maurel B., Combescure A., "An SPH shell formulation for plasticity and fracture analysis in explicit dynamics", International Journal For Numerical Methods In Engineering, 2008, p. $949-971$.

Monaghan J.J., “An introduction to SPH”, Comput. Phys., Commun., 48, 1988, p. 89-96.

Monaghan J.J., Lattanzio J.C., "A refined particle method for astrophysical problems", Astronomy and Astrophysics, 1985, 149, p. 135-143.

Newmark N.M., "A method of computation for Structural Dynamics”, Journal of Engineering Mechanics Division, ASCE, 1959, vol. 85, p. 67-94.

Perzyna P., "Fundamental problems in viscoplasticity, Recent Advances in Applied Mechanics", Academic press, New York, 1966, 9, p. 243-377. 
Potapov S., Maurel B., Combescure A., Fabis J., "Modeling accidental-type fluid-structure interaction problems with the SPH method", Computers and Structures, vol. 87, 2009, p. 721-734.

Rabczuk T., Belytschko T., Xiao S. P., "Stable particle methods based on Lagrangian kernels”, Comput. Methods. Appl. Mech. Engrg, 2004, 193, p. 1035-1063.

Randles P. W., Libersky L. D., "Recent improvements in SPH modelling of hypervelocity impact”, Int. J. Impact Engrg., 1997, 20, p. 525-532.

Randles P.W., Libersky L.D., "Normalized SPH with stress points", Int. J. Numer. Meth. Engng., 2000, 48, p. 1445-1462.

Réthoré J., Gravouil A., and Combescure A., "A combined space-time extended finite element method”, Int. J. Numer. Meth. Engng., 2005, 64, p. 260-284.

Simo J.C., Tarnow N, Wong KK., "Exact energy-momentum conserving algorithms and symplectic schemes for nonlinear dynamics", Computer Methods in Applied Mechanics and Engineering, 1992, vol. 100, n ${ }^{\circ} 1$, p. 63-116.

Swegle J.W., Hicks D.A., Attaway S.W., "Smooth particle hydrodynamics stability analysis", J. Comput. Phys., 116, 1995, p. 123-134.

Xiao S.P., Belytschko T., "Material stability analysis of particle methods", Adv. Comput. Math., 23, 2005, p. 171-190.

Zienkiewicz O.C., Taylor R.L., The Finite Element Method ( $5^{\text {th }}$ Edition) vol 1, The Basis, Butterword-Heinemann, Oxford, 2000.

Received: 10 October 2010

Accepted: 25 February 2011 